\title{
A Comparative Transcriptomic and Proteomic Analysis of Hexaploid Wheat's Responses to Colonization by Bacillus velezensis and Gaeumannomyces graminis, Both Separately and Combined
}

\author{
Xingxing Kang, ${ }^{1}$ Lanhua Wang, ${ }^{1}$ Yu Guo, ${ }^{1}$ Muhammad Zain ul Arifeen, ${ }^{1}$ Xunchao Cai, ${ }^{1}$ Yarong Xue,,${ }^{1,+}$ \\ Yuanqin $\mathrm{Bu}^{2}{ }^{2}$ Gang Wang, ${ }^{3}$ and Changhong Liu, ${ }^{1,+}$ \\ ${ }^{1}$ State Key Laboratory of Pharmaceutical Biotechnology, School of Life Sciences, Nanjing University, Nanjing, China \\ ${ }^{2}$ Nanjing Institute of Environmental Sciences, Key Laboratory of Pesticide Environmental Assessment and Pollution Control, \\ Ministry of Ecology and Environment, Nanjing, China \\ ${ }^{3}$ State Key Laboratory of Cotton Biology, School of Life Sciences, Henan University, Kaifeng, Henan, China
}

Accepted 23 May 2019.

\begin{abstract}
Tritrophic interactions involving a biocontrol agent, a pathogen, and a plant have been analyzed predominantly from the perspective of the biocontrol agent. To explore the adaptive strategies of wheat in response to beneficial, pathogenic, and combined microorganisms, we performed the first comprehensive transcriptomic, proteomic, and biochemical analysis in wheat roots after exposure to Bacillus velezensis CC09, Gaeumannomyces graminis var. tritici, and their combined colonization, respectively. The transcriptional or translational programming of wheat roots inoculated with beneficial $B$. velezensis showed mild alterations compared with that of pathogenic $G$. graminis var. tritici. However, the combination of $B$. velezensis and $G$. graminis var. tritici activated a larger transcriptional or translational program than for each single microorganism, although the gene expression pattern was similar to that of individual infection by $G$. graminis var. tritici, suggesting a prioritization of defense against $G$. graminis var. tritici infection. Surprisingly, pathogen-associated molecular pattern-triggered immunity and effector-triggered immunity made wheat pretreated with $B$. velezensis more sensitive to subsequent $G$. graminis var. tritici infection. Additionally, B. velezensis triggered a salicylic acid (SA)-dependent mode of induced systemic resistance that resembles pathogen-induced systemic acquired resistance. Wheat plants mainly depend on SA-mediated resistance, and not that mediated by jasmonic acid (JA), against the necrotrophic pathogen $G$. graminis var. tritici. Moreover, SA-JA interactions resulted in antagonistic effects regardless of the type of microorganisms in wheat. Further enhancement of SA-dependent defense responses such as lignification to the combined infection was shown to reduce
\end{abstract}

${ }^{\dagger}$ Corresponding authors: C. Liu; chliu@nju.edu.cn and

Y.Xue; xueyr@nju.edu.cn

Funding: The research is financially supported by the National Natural Science Foundation of China (31471810, 41773083, and 31272081) and the National Key R \& D Project of China (2017YFD0800705).

*The $\boldsymbol{e}$-Xtra logo stands for "electronic extra" and indicates that five supplementary figures and seven supplementary tables are published online.

The author(s) declare no conflict of interest.

๑) 2019 The American Phytopathological Society the level of induced JA-dependent defense against subsequent infection with $G$. graminis var. tritici. Altogether, our results demonstrate how the hexaploid monocot wheat responds to beneficial or pathogenic microorganisms and prolongs the onset of take-all disease through modulation of cell reprogramming and signaling events.

Keywords: bacterium-plant symbiosis, biocontrol bacteria, fungusplant interactions, genomics, induced systemic resistance, metabolomics, molecular signaling, omics, plant defense system, plant disease, plantmicrobial interaction, phytopathology, proteomics

Wheat is the second most important food crop globally, serving as a staple food for much of the world's population (Singh et al. 2011; Singh et al. 2018). Global wheat production is severely affected by "take-all", a major fungal disease caused by Gaeumannomyces graminis var. tritici, which can cause significant yield and economic losses and remains challenging to control (Bithell et al. 2016). G. graminis var. tritici is a necrotrophic soilborne pathogen that infects wheat roots via hyphae by penetrating root cortical cells and progressing into the stem, where it mainly disrupts water flow, thus causing stunted growth and premature death of the plant. This fungal disease is not restricted to wheat but is also found in many other plants such as barley, triticale, and rye (Yang et al. 2015a). To control the disease and reduce yield loss, considerable effort has been made to elucidate the underlying mechanism of G. graminis var. tritici pathogenicity and its interaction with the host (Daval et al. 2011; Puga-Freitas et al. 2017; Yang et al. 2015b). The molecular mechanisms involved in plant response to pathogenic fungi are relatively well documented for fungi such as Botrytis cinerea, Fusarium oxysporum, Parastagonospora nodorum, and Zymoseptoria tritici (BerrocalLobo and Molina 2008; Duba et al. 2018; Williamson et al. 2007). In contrast, very little is known about the molecular response of wheat to $G$. graminis var. tritici infection. From previous transcriptomic studies, it was suggested that several genes involved in signal transduction, including pathogenassociated molecular patterns (PAMPs), are differentially expressed in $G$. graminis var. tritici-infected wheat as compared with controls (Guilleroux and Osbourn 2004; PugaFreitas et al. 2017). 
Currently, control of take-all mainly relies on cultural practices such as crop rotation with nonhost plants and utilization of ammonium fertilizers to make the wheat rhizosphere unfavorable to G. graminis var. tritici (Cook 2003). However, due to the absence of plant genetic resistance and efficient fungicides against the disease, it is increasingly believed that the application of biological control agents such as Bacillus subtilis, Bacillus velezensis, and Pseudomonas fluorescens may provide alternative management strategies to control the disease (Daval et al. 2011; Kang et al. 2018; Yang et al. 2015a). Our previous research demonstrated that $B$. velezensis $\mathrm{CC} 09$, an endophytic biocontrol bacterium, has unique disease prevention capability. It not only can lead to the production of broad-spectrum antifungal metabolites but also directly inhibits fungal growth in vitro and in vivo, and can be transmitted in plants, through stalks, from root to leaf. Like a "vaccine", it can be applied once on the root to induce plant resistance to control both root take-all and leaf spot blotch (Bipolaris sorokiniana) diseases at the same time (Cai et al. 2017; Kang et al. 2018). However, the molecular mechanism of $B$. velezensis-mediated resistant against fungal disease in wheat is still unknown.

Despite genome complexity, several experiments have been conducted to understand possible response mechanisms of wheat challenged with beneficial bacteria (i.e., Azospirillum brasilense and $B$. amyloliquefaciens) or pathogenic fungi (i.e., Botrytis cinerea, F. oxysporum, and G. graminis var. tritici), and a number of genes or proteins involved in diverse plant defense responses mediated by salicylic acid (SA), jasmonic acid (JA), or ethylene (ET) have been identified using transcriptomic and proteomic techniques (Berrocal-Lobo and Molina 2008; Erayman et al. 2015; Puga-Freitas et al. 2017; Saia et al. 2015; Souza et al. 2014; Srivastava et al. 2016; Williamson et al. 2007; Xin et al. 2012; Zhang et al. 2018). However, to date, no study has been performed to understand the precise molecular mechanism of wheat in response to $B$. velezensis. Furthermore, current knowledge regarding the response of plants to either beneficial or pathogenic infections is only based on studying the interaction between plants and microbes using single host-single strain model systems rather than host-multiple microbe systems (Engelmann et al. 2011; Gonzalez et al. 2018). No studies have been carried out to understand the mechanism of wheat response to both beneficial and pathogenic microorganisms, a scenario more akin to what is found in natural agriculture ecosystems.

Plants employ diverse defense mechanisms at local or systemic levels to modify the environments in which they live. The first line of defense, defined as structural constitutive resistance, is mediated by physical barriers (i.e., cuticle, suberin deposition, and cell wall lignification) and the production of antagonistic chemicals (Jain et al. 2018; Piasecka et al. 2015). The second line of defense comprises systemic acquired resistance (SAR) and induced systemic resistance (ISR), mediated by the plant innate immune system against a variety of pathogens. SAR is a pathogen-induced defense system activated by many endogenous signals such as SA, pipecolic acid, N-hydroxy-pipecolic acid, and azelaic acid/glycerol 3-phosphate in plants upon pathogen priming (Chen et al. 2018; Hartmann et al. 2018; Klessig et al. 2018; Spoel and Dong 2012). In contrast to SAR, ISR is initiated by beneficial microbial priming (i.e., endophytes or plant-growth-promoting rhizobacteria [PGPR]), which triggers JA- and ET-mediated signaling pathways and enhances disease resistance against pathogen attack (Gond et al. 2015; Jain et al. 2018; Pieterse et al. 2014). As such, JA- or ET-mediated ISR is also the main mechanism for indirect control of disease by biocontrol agents (Bano and Muqarab 2017; Mhlongo et al. 2018; Stringlis et al. 2018). Thus, ISR offers prospect as a plant protection system against pathogens to be used in modern agriculture management systems to at least reduce the amount of chemical pesticides. However, it is not yet clear whether SAR or ISR plays a more important role in disease control when plants are cocolonized by biocontrol bacteria and pathogens. Furthermore, it remains to be determined whether the structural constitutive resistance of plants can be enhanced by colonization of biocontrol bacteria to reduce the damage caused by pathogens.

B. velezensis $\mathrm{CC} 09$ was an endophytic biocontrol bacterium isolated from the healthy Cinnamomum camphora leaf tissue (Cai et al. 2016). Our previous studies showed that $B$. velezensis CC09 has the ability to colonize almost every part of wheat root, including the epidermis, cortex, and xylem vessels, and even migrated to stems and leaves, resulting in $66.67 \%$ diseasecontrol efficacy (DCE) of take-all and 21.64\% DCE of spot blotch (Kang et al. 2018). The aim of this follow-up study was to elucidate the molecular mechanism in wheat roots of the response to a $B$. velezensis, a take-all-causing pathogen G. graminis var. tritici, and their combined colonization using RNA-seq, iTRAQ (isobaric tags for relative and absolute quantification), and biochemical analysis techniques. The possible synergistic interaction between $B$. velezensis and G. graminis var. tritici inside root tissues and its potential effect on the defense response system of wheat were explored in detail.

\section{RESULTS}

\section{Transcriptional profiles of wheat in response \\ to $B$. velezensis, G. graminis var. tritici, and}

B. velezensis + G. graminis var. tritici colonization.

Screening of differentially expressed genes. The RNA-seq data were well validated by quantitative real-time PCR (qRTPCR) analysis, indicating that the data could be used for the follow-up analysis (Supplementary Table S1; Supplementary Fig. S1). A comparative transcriptomic analysis was undertaken to explore the molecular mechanism of wheat roots in response to the biocontrol bacterium $B$. velezensis, the takeall pathogen $G$. graminis var. tritici, and their combination (B. velezensis + G. graminis var. tritici). In general, the expression of most genes remained unchanged among the total of 80,566 detected genes (reads per kilobase per million [RPKM > $0)$. In total, 10,780 genes (4,831 upregulated and 5,945 downregulated), $13.4 \%$ of the total expressed genes, were identified as differentially expressed genes (DEGs) caused by the combination of $B$. velezensis and $G$. graminis var. tritici (Supplementary Table S2). In contrast, only 3,127 (1,355 upregulated and 1,772 downregulated) (3.9\% of the total expressed genes) and 5,678 (2,833 upregulated and 2,845 downregulated) (7.0\% of the total expressed genes) DEGs were identified in the $B$. velezensis- and $G$. graminis var. tritici-colonized wheat roots, respectively (Supplementary Fig. S2). The number of DEGs caused by G. graminis var. tritici infection was 1.8 times that of the B. velezensis-colonized sample. However, the number of DEGs in combination-colonized samples was 3.4 and 1.9 times those of the B. velezensis- and G. graminis var. tritici-colonized samples, respectively. Moreover, both the upand downregulated DEGs of wheat roots that were cocolonized by $B$. velezensis and $G$. graminis var. tritici were much higher than those of the B. velezensis- or G. graminis var. tritici-colonized wheat roots. For instance, the number of upregulated DEGs of the cocolonized sample was 3.6 and 1.7 times those of the B. velezensis- and G. graminis var. tritici-colonized samples, respectively; the number of downregulated DEGs of the cocolonized sample was 3.4 and 2.1 times higher than those of the B. velezensis- and G. graminis var. tritici-colonized samples, respectively. Meanwhile, the number of up- and downregulated 
DEGs of G. graminis var. tritici-colonized wheat roots was 2.1 and 1.6 times those of $B$. velezensis-colonized wheat roots, respectively. In addition, a statistical analysis revealed that, when wheat was subjected to G. graminis var. tritici or the combined infection, the expression level of most genes were induced with a higher number of extreme transcriptional changes (>10-fold) compared with B. velezensis treatment. In conclusion, changes in gene expression levels were more drastic when wheat was challenged by two microorganisms than when treated with a single microorganism, especially for the beneficial microorganism.

Clustering of DEGs. Hierarchical cluster analysis was conducted using 13,400 DEGs that showed significant differential expression in at least one sample (Fig. 1). The analysis clearly revealed that the endophytic $B$. velezensis-colonized root sample and the control samples clustered into one branch and the pathogenic G. graminis var. tritici- and combinationcolonized samples clustered into another branch. Cluster analysis of the RPKM data showed that the gene expression pattern could be divided into seven clusters based on the expression levels of the DEGs in B. velezensis-, G. graminis var. tritici-, and combination-colonized roots versus the microbefree control (Supplementary Table S3); cluster D was enriched in transcripts that showed large fold changes in expression between $B$. velezensis-colonized and control roots, while cluster $\mathrm{G}$ represented the fold changes between $G$. graminis var. tritici-colonized and control roots. Clusters $\mathrm{E}$ and $\mathrm{F}$ contained DEGs with very high expression levels in combinationcolonized roots compared with the control.

Discrete transcriptome responses to bacterial or fungal colonization. The short time-series expression miner (STEM) platform was used to analyze the transcriptome dynamics in response to B. velezensis, G. graminis var. tritici, and combination colonization on total DEGs. In total, four important temporal gene expression profiles were obtained (Fig. 2A). Most of the upregulated genes $(2,278)$ were clustered into profile 17 , and many DEGs in this profile are involved in the biosynthesis of secondary metabolites (i.e., terpenoid backbone, diterpenoid, stilbenoid/diarylheptanoid/gingerol, and flavonoid biosynthesis) as well as other metabolic pathways associated with plant defense such as the plant-pathogen interaction pathway (Supplementary Figs. S3 and S4). Genes encoding pathogenesis-related (PR) proteins and pattern recognition receptors (PRRs) involved in PAMP-triggered immunity (PTI), calcium influx genes, hormone regulation genes, respiratory burst oxidase genes, and mitogen-activated protein kinase cascade genes were all enhanced in response to $B$. velezensis, $G$. graminis var. tritici, and both combined. Based on the predicted nucleotide-binding site leucine-rich repeat (NBS-LRR) family genes in the wheat genome (Peng and Yang 2017), we conducted further analysis of the DEGs encoding NBS-LRR proteins in B. velezensis-, G. graminis var. tritici-, and combination-colonized wheat roots. The distribution of up- and downregulated NBS-LRR family DEGs was calculated and presented in a Venn diagram (Fig. 2B). Expression of 13 such DEGs was elevated post B. velezensis inoculation, after which it returned to normal, while the levels of 46 additional DEGs increased following treatment with combination. In conclusion, the combined interaction of $B$. velezensis and G. graminis var. tritici elicited more significant changes in DEGs involved in both PTI and effector-triggered immunity (ETI) defense programs than that of each single microorganism.

Profile 4 contained the most transcripts $(3,368)$; most of genes in this profile as well as profiles 1 and 10 were largely involved in primary metabolism such as the metabolism of carbohydrates (i.e., glyoxylate, dicarboxylate, galactose, starch, and sucrose), lipids, amino acids, and photosynthesis, and these genes were dynamically downregulated. The upregulation of genes involved in wheat defense pathways and the downregulation of genes involved in primary metabolism indicate that readjustment of plant primary metabolism likely contributes to plant defense responses against pathogen attack.

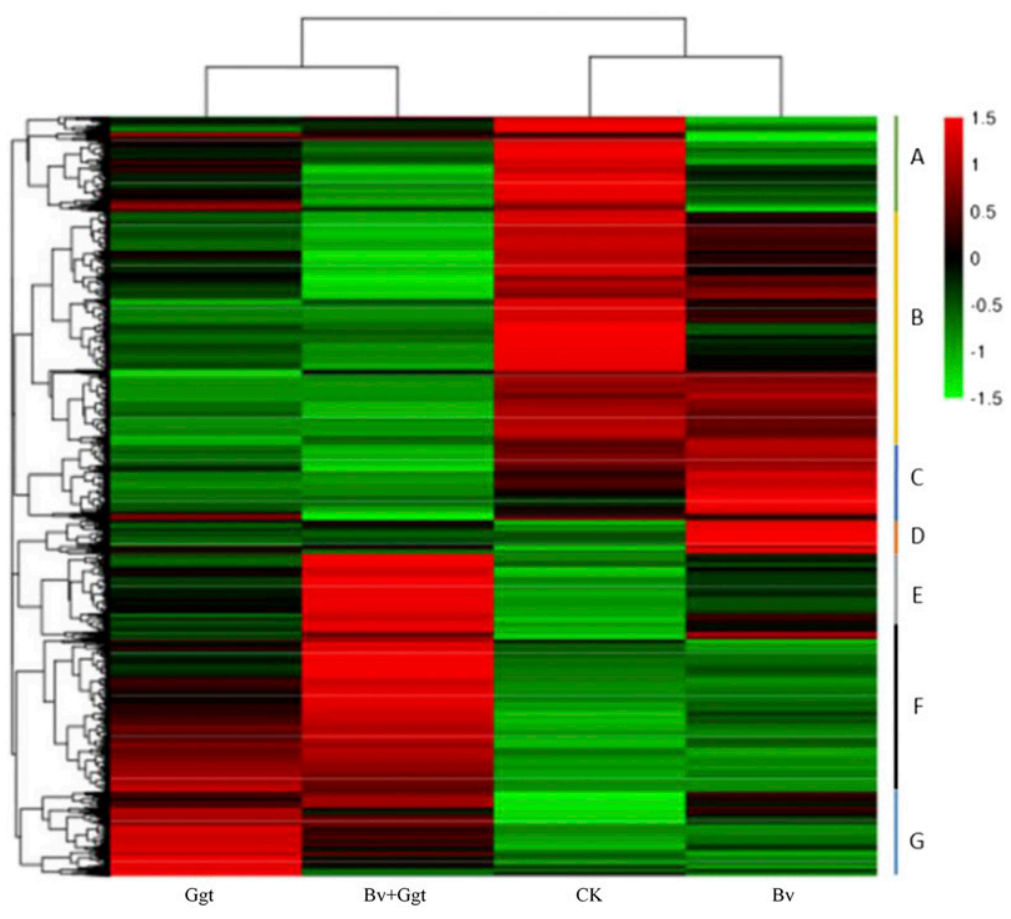

Fig. 1. Hierarchical clustering analyses of differentially expressed genes. According to the three sample's reads per kilobase per million, red indicates high expression of the gene, green indicates low expression of genes. Bv, Ggt, and Bv+Ggt = Bacillus velezensis, Gaeumannomyces graminis var. tritici, and their combination, respectively. 
Expression of genes encoding JA and SA synthases. JA is a major phytohormone induced by plants to elicit systemic resistance against pathogens. Lipoxygenases (LOX), allene oxide synthase, allene oxide cyclase, and oxo-phytodienoate reductase (OPR) are the main enzymes involved in the biosynthesis of JA (Supplementary Fig. S5). In this study, eight DEGs involved in LOX biosynthesis were identified, and $87.5 \%$ of these genes were upregulated in $B$. velezensis-colonized sample; four LOX genes were found in the G. graminis var. tritici-colonized sample and $75.0 \%$ of these genes were upregulated; and six LOX genes were identified in the combination-colonized sample but only $33.3 \%$ of them were upregulated (Table 1). In other words, most of the LOX genes were downregulated in the combination-colonized sample. A similar regulatory pattern of DEGs was found for the OPR genes.

Phenylalanine ammonia lyase (PAL) is a key enzyme involved in SA biosynthesis. In total, 13 DEGs encoding PAL enzymes were identified and all of these genes were upregulated in the pathogenic G. graminis var. tritici-colonized roots; 2 were found in the combination-colonized sample, with all upregulated; and 3 were identified in the $B$. velezensis-colonized sample, with all downregulated (Table 1). These data suggest that the biocontrol bacterium $B$. velezensis might have the ability to stimulate JA production in wheat plants, whereas the pathogen $G$. graminis var. tritici might induce the accumulation of JA and SA. These results also suggest that JA content might be reduced whereas SA content might be increased in wheat cocolonized by $B$. velezensis and $G$. graminis var. tritici.

Expression of JA- and SA-responsive genes in wheat roots. The plant hormones JA and SA are important regulators of induced defense mechanisms. We next sought to determine how the defense pathways mediated by JA and SA respond in B. velezensis-, G. graminis var. tritici-, or combinationcolonized samples. $P R$ protein $1(P R I)$ and $P D F 1.2$, marker resistance genes representing SA and JA pathways, respectively, were investigated (Vos et al. 2015). The transcriptome data demonstrated that, regardless of whether the wheat roots were colonized by a single species or two combined, or a beneficial or pathogenic microorganism, $P R 1$ genes were always upregulated (Table 2). In all, 12 and $13 P R 1$ genes were significantly changed for $G$. graminis var. tritici and combination samples, respectively. In contrast, only six PRI DEGs were identified in B. velezensis-colonized samples. Additionally, in the G. graminis var. tritici and combination samples, there were four and five PRl genes with an approximately four- to sevenfold change,

Table 1. Profile of gene expression (up/down) involved in the biosynthesis of jasmonic acid (JA) and salicylic acid (SA) in wheat roots

\begin{tabular}{lcccc}
\hline $\begin{array}{l}\text { Plant } \\
\text { hormone }\end{array}$ & $\begin{array}{c}\text { Gene } \\
\text { name }^{\mathbf{a}}\end{array}$ & $\begin{array}{c}\text { Bacillus } \\
\text { velezensis }\end{array}$ & $\begin{array}{c}\text { Gaeumannomyces } \\
\text { graminis } \\
\text { var. tritici }\end{array}$ & $\begin{array}{c}\text { Both organisms } \\
\text { combined }\end{array}$ \\
\hline JA & LOX & $7 / 1$ & $3 / 1$ & $2 / 4$ \\
JA & AOS & $1 / 0$ & - & $0 / 2$ \\
JA & AOC & - & - & $1 / 2$ \\
JA & OPR & $5 / 3$ & $7 / 2$ & $5 / 7$ \\
SA & PAL & $0 / 3$ & $13 / 0$ & $2 / 0$ \\
\hline
\end{tabular}

${ }^{\mathrm{a}} \mathrm{LOX}=$ lipoxygenases, $\mathrm{AOS}=$ allene oxide synthase, $\mathrm{AOC}=$ allene oxide cyclase, OPR = oxo-phytodienoate reductase, and PAL = phenylalanine ammonia lyase; - indicates that expression of the genes was not detected.

Table 2. Expression of maker resistance genes (up/down) that can represent jasmonic acid (JA)- and SA-mediated defense pathways in Bacillus velezensis-, Gaeumannomyces graminis var. tritici-, and combinationcolonized roots

\begin{tabular}{lccc}
\hline Gene name $^{\text {a }}$ & B. velezensis & G. graminis var. tritici & Combination \\
\hline$P R 1$ & $6 / 0$ & $12 / 0$ & $13 / 0$ \\
$P D F 1.2$ & $0 / 1$ & $0 / 5$ & $0 / 6$ \\
\hline
\end{tabular}

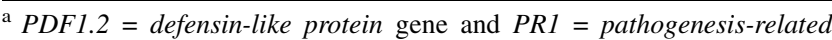
protein 1 gene.

A

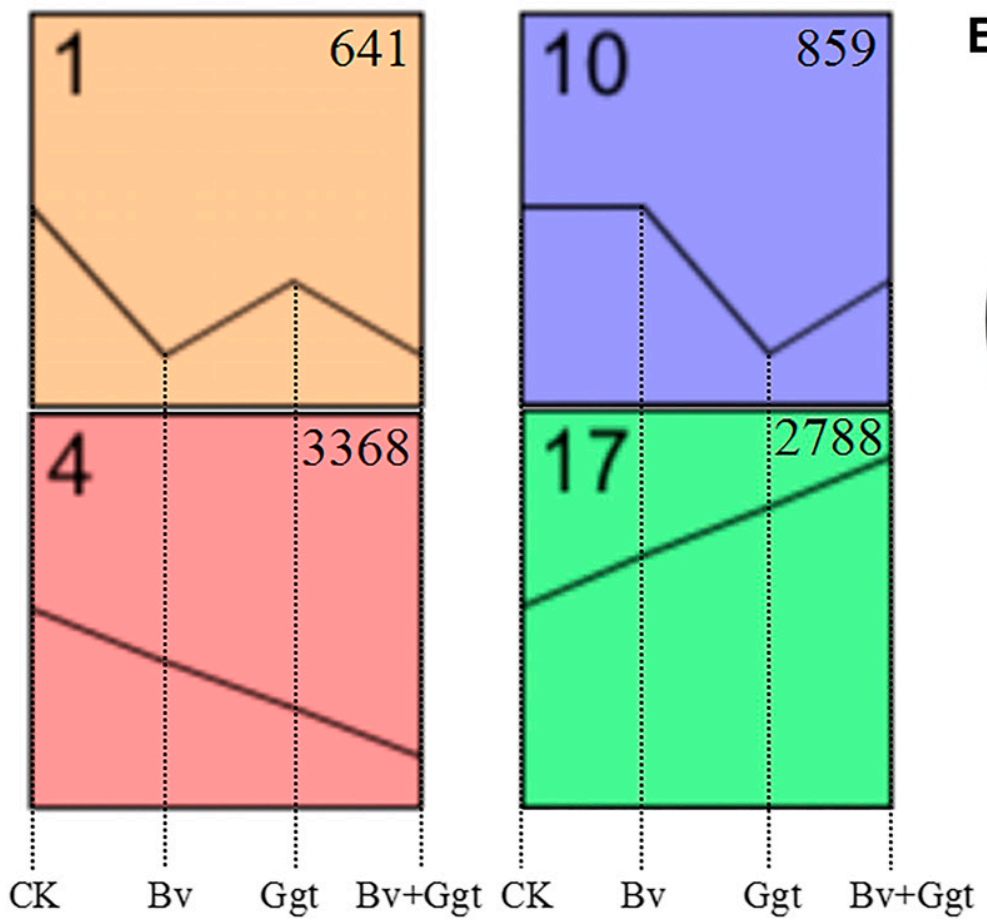

B $\quad \mathrm{Bv} \quad$ Ggt

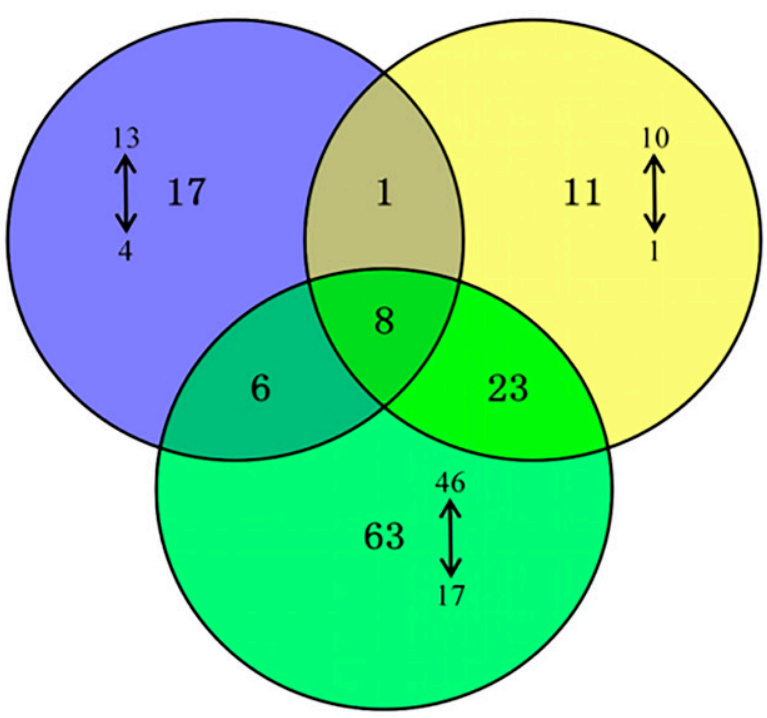

$\mathrm{Bv}+\mathrm{Ggt}$

Fig. 2. A, Expression patterns and B, numbers of differentially expressed genes encoding nucleotide-binding site leucine-rich repeat (NBS-LRR) proteins. Expression patterns of genes identified by transcriptome comparisons of treatments with Bacillus velezensis (Bv), Gaeumannomyces graminis var. tritici (Ggt), both combined $(\mathrm{Bv}+\mathrm{Ggt})$, and the control $(\mathrm{CK})$. The Venn diagram illustrates the numbers of NBS-LRR family genes with upregulated (upward-pointing arrow) or downregulated (downward-pointing arrow) expression in B. velezensis-, Gaeumannomyces graminis var. tritici-, and combination-colonized wheat roots. 
respectively, whereas, in the $B$. velezensis sample, there was a single $P R I$ gene with an approximately one- to twofold change (Supplementary Table S4). When taking into account changes in SA content (Fig. 3), these results indicate that the SAmediated resistance pathway is induced regardless of whether or not wheat roots are colonized by beneficial bacteria, pathogenic fungi, or both but the presence of the pathogen (G. graminis var. tritici sample) enhances this pathway and the combined interaction (combination sample) further induces it.

In contrast to the expression of $P R I$ genes, the expression of the JA-signaling pathway marker $P D F 1.2$ gene was inhibited in all treatment groups (Table 2). Of the PDF1.2 genes, only one (Traes_4DL_909858768) showed a significant (1.08-fold) change caused by the beneficial $B$. velezensis. In contrast, $60 \%$ (3 of 5) of PDF1.2 genes showed an approximately three- to fourfold change in the $G$. graminis var. tritici sample, while $83 \%$ (5 of 6) showed an approximately three- to fourfold change in the combination sample. In summary, compared with $G$. graminis var. tritici infection, the expression of JAresponsive genes was attenuated in the combination sample.

Expression of genes involved in lignin biosynthesis. Deposition of lignin is thought to contribute to plant resistance by preventing the diffusion of pathogens (Bellincampi et al. 2014). To understand how lignification changes in response to single or combined, beneficial or pathogenic microorganisms in wheat roots, we next explored the expression changes of key genes involved in lignin biosynthesis. Based on previous studies, PAL, cinnamate 4-hydroxylase $(\mathrm{C} 4 \mathrm{H})$, and 4-coumarate CoA ligase (4CL) catalyze the first three steps in the phenylpropanoid pathway (Fig. 4A), producing precursors to lignin. Whereas genes encoding these enzymes were downregulated in the $B$. velezensis sample, all were found to be induced upon G. graminis var. tritici treatment (Fig. 4B). In the combination sample, although most of the genes encoding 4CL protein were downregulated, critical lignin biosynthesis genes encoding PAL and $\mathrm{C} 4 \mathrm{H}$ were upregulated. This result indicated that precolonization of $B$. velezensis might lead to an increase of cell wall lignification, or it is possible that the interaction between $B$. velezensis and G. graminis var. tritici triggered the complex regulation of gene expression in planta.

Proteome profiles of wheat in response to $\mathrm{B}$. velezensis, G. graminis var. tritici, and combination colonization.

To identify the differentially expressed proteins (DEPs) in wheat roots colonized by $B$. velezensis, G. graminis var. tritici, and both combined relative to control plants, we performed a quantitative proteomic analysis based on iTRAQ technology. According to the standards of DEP identification $(\geq 1.5$-fold cutoff, $P$ value $<0.05)$, only eight proteins, no matter whether in $G$. graminis var. tritici- or in combination-colonized wheat roots, showed a significant difference from 783 detected proteins (Supplementary Table S5). However, no DEPs were identified in the B. velezensis-colonized wheat roots. Among these DEPs, five were regulated in both G. graminis var. triticiand combination-colonized roots, including phosphoglycerate kinase, phosphoenolpyruvate carboxylase 2 , aspartate aminotransferase, cyclophilin A, and a predicted protein (Supplementary Table S6). Three DEPs were specifically regulated in

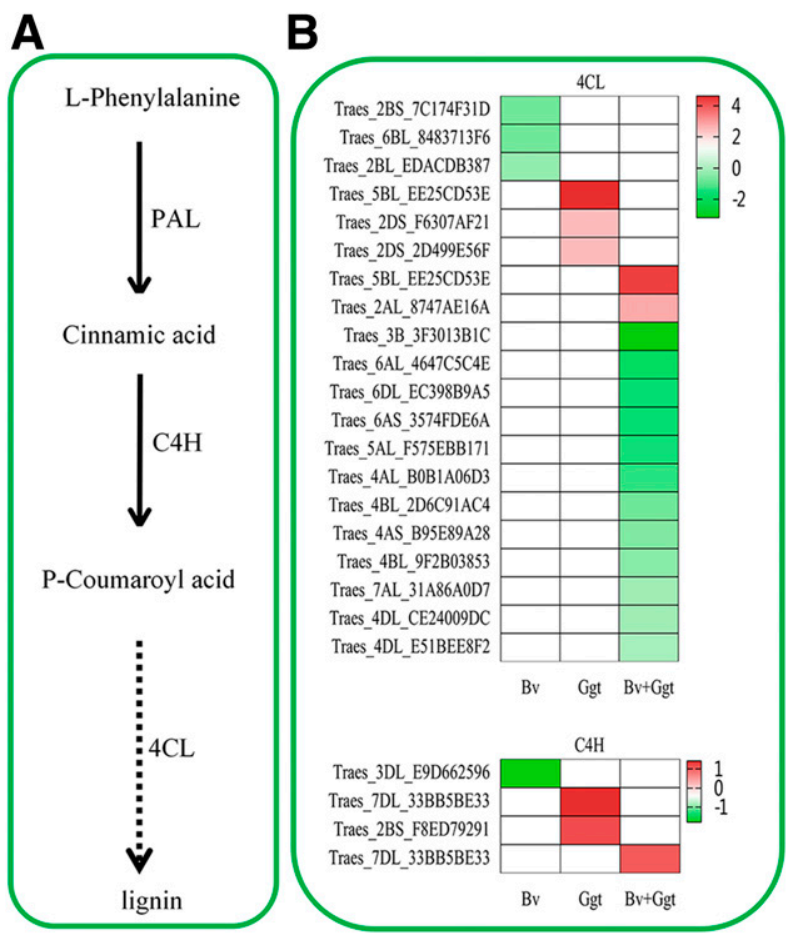

Fig. 4. Effects of Bacillus velezensis (Bv), Gaeumannomyces graminis var. tritici (Ggt), and both combined (Bv+Ggt) on the biosynthesis of lignin. A, Overview of lignin biosynthesis. B, Heat map of lignin biosynthesis-related gene expression. $\mathrm{PAL}=$ phenylalanine ammonia lyase, $\mathrm{C} 4 \mathrm{H}=$ cinnamate 4-hydroxylase, and 4CL = 4-coumarate CoA ligase.
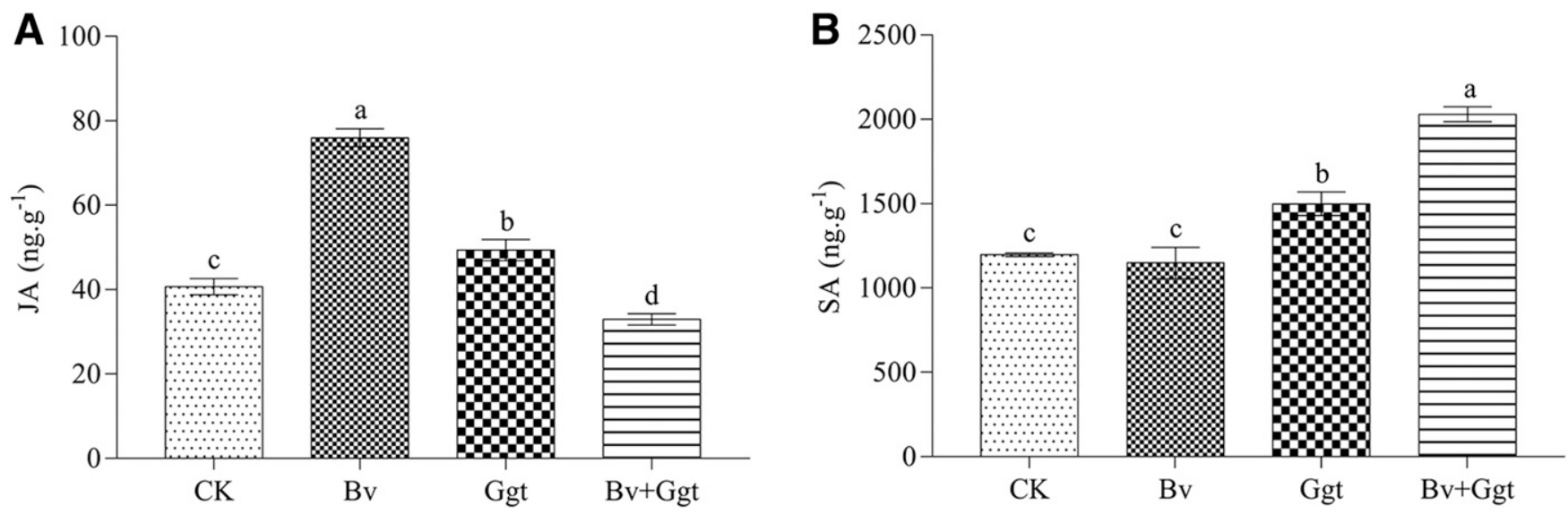

Fig. 3. Effects of Bacillus velezensis (Bv), Gaeumannomyces graminis var. tritici (Ggt), and both combined (Bv+Ggt) on the biosynthesis of jasmonic acid (JA) and salicylic acid (SA). A, JA and B, SA concentration in wheat roots. Different letters indicate a significant difference between treated and control (CK) samples based on Fisher's least significant difference test at $P$ value $=0.05$. 
G. graminis var. tritici-colonized wheat roots, including putative $26 \mathrm{~S}$ proteasome non-ATPase regulatory subunit $3,40 \mathrm{~S}$ ribosomal protein S16, and Serpin-ZX. Likewise, three DEPs were specifically regulated in combination-colonized roots, including endoplasmin-like protein, 40S ribosomal protein S6, and serine hydroxymethyltransferase, which might contribute to wheat resistance improvement.

Comparison analysis of transcriptome and proteome data.

In total, 129 proteins were identified for which corresponding transcripts were represented in the RNA-seq data. The comparison analyses of transcript and protein data from B. velezensis, G. graminis var. tritici, and combination treatments revealed that the Pearson correlation $(r)$ was 0.0196 , 0.0927, and 0.0116, respectively (Fig. 5).

\section{Contents of phytohormones and lignin in wheat roots.}

To validate the regulatory effect of $B$. velezensis, G. graminis var. tritici, and combination colonization on the biosynthesis of phytohormones, we measured the concentrations of JA and SA in wheat roots (Fig. 3). Compared with that of controls (40.69 $\mathrm{ng} \mathrm{g}^{-1}$ ), the amount of JA was significantly increased in B. velezensis-colonized $\left(75.97 \mathrm{ng} \mathrm{g}^{-1}\right)$ and $G$. graminis var. tritici-colonized (49.37 $\mathrm{ng} \mathrm{g}^{-1}$ ) wheat roots but decreased in the combination-colonized roots $\left(32.95 \mathrm{ng} \mathrm{g}^{-1}\right)$. In contrast, the concentration of SA was not significantly altered in B. velezensis-colonized roots $\left(1,148.52 \mathrm{ng} \mathrm{g}^{-1}\right)$ but was significantly increased in $G$. graminis var. tritici-colonized $\left(1,499.90 \mathrm{ng} \mathrm{g}^{-1}\right)$ and combination-colonized $\left(2,030.24 \mathrm{ng} \mathrm{g}^{-1}\right)$ samples relative to that of the control $\left(1,197.32 \mathrm{ng} \mathrm{g}^{-1}\right)$. These results are consistent with the data obtained from the transcriptomic studies (Table 1).

Lignin is an aromatic polymer that is mainly deposited in secondary thickened cell walls, where it provides strength and imperviousness against pathogen infection. To understand the role of lignin in response to colonization of single or combined, beneficial or pathogenic microorganisms, lignin content was investigated. A comparison of wheat root cross-sections demonstrated that inoculation of B. velezensis, G. graminis var. tritici, or both combined resulted in more lignified tissue compared with noninoculated plants (Fig. 6). However, lignin content was increased in the presence of the pathogen (Fig. 6C and D), with the highest levels observed in the combination sample, suggesting that the coinfection by B. velezensis and $G$. graminis var. tritici further strengthens the secondary wall thickness.

\section{DISCUSSION}

This is the first comprehensive report of wheat, a hexaploid plant, aiming to analyze changes in transcription and translation in response to single or combined, beneficial or pathogenic microorganisms. Based on the current study, compared with the G. graminis var. tritici and combination samples, there were not only fewer DEGs or DEPs but also relatively mild gene expression levels on the whole in the B. velezensis sample, especially for the plant-pathogen interaction pathway genes, NBS-LRR family genes, and PR1 proteins. Downregulation of root immune responses has also been described for ISRinducing PGPR such as B. subtilis FB17 and P. fluorescens WCS417r (Millet et al. 2010; Verhagen et al. 2004). Lakshmanan et al. (2013) suggested that beneficial microbes may partially block immune responses in Arabidopsis thaliana roots and support efficient root colonization and establishment of rhizosphere microbial communities. To achieve similar effects and establish a mutualistic interaction with the plant, beneficial $B$. velezensis may therefore need to prevent, weaken, or overcome host immune responses in wheat roots. Our results showed that combined inoculation of wheat roots elicited more large-scale transcriptional changes, especially for genes associated with plant-interaction pathways that are closely related
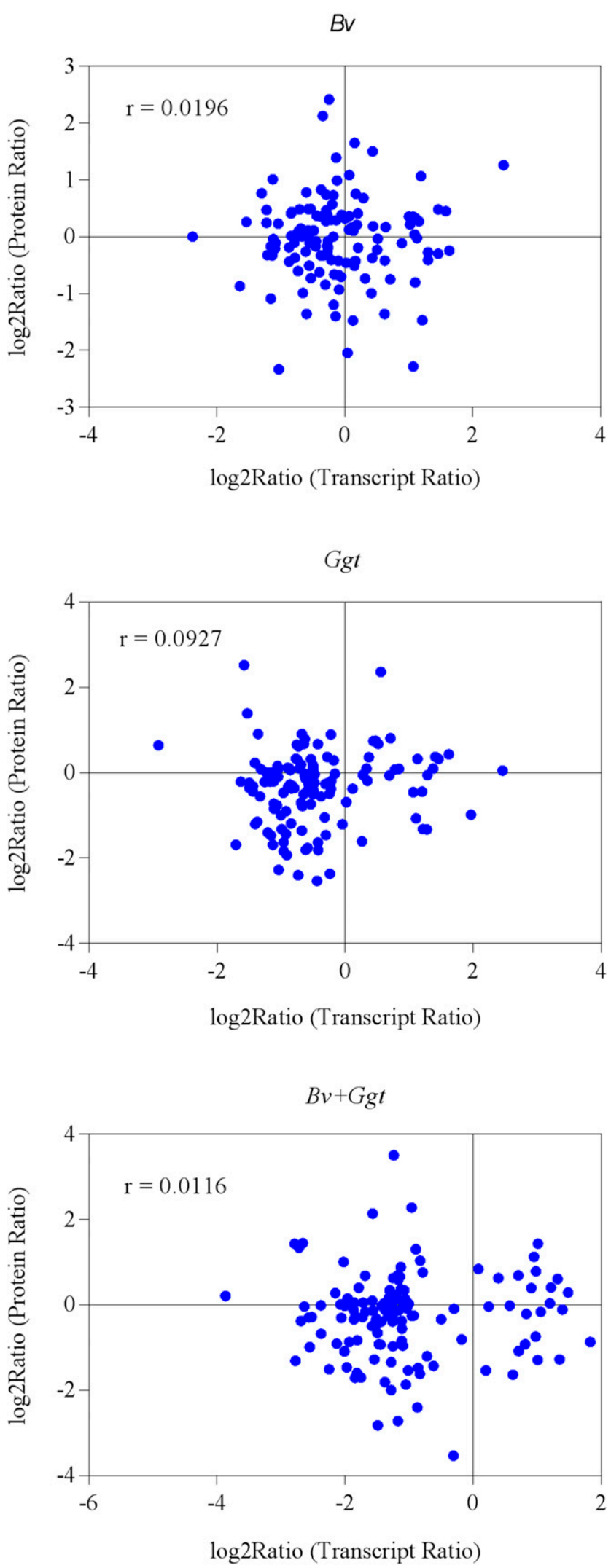

Fig. 5. Correlation graph of transcripts and associated proteins. Bv, Ggt, and $\mathrm{Bv}+\mathrm{Ggt}=$ Bacillus velezensis, Gaeumannomyces graminis var. tritici, and their combination, respectively. 
to plant defense. According to the hypothesis of trade-off between constitutive and inducible resistance traits in plants (Morris et al. 2006), a wheat plant having more potential to induce DEGs may have improved resistance. Based on the current data, we also discovered that $B$. velezensis triggered a PTI-mediated defense system in wheat roots, which is consistent with previous studies (Lopez-Gomez et al. 2012; Trdá et al. 2014). To our knowledge, however, the mechanisms that allow beneficial bacteria to colonize plants are still limited. A few studies on interactions between Burkholderia phytofirmans and A. thaliana (Trdá et al. 2014) and Sinorhizobium meliloti and Lotus japonicus (Lopez-Gomez et al. 2012) have demonstrated that these PGPR strains triggered PTI in a manner dependent on flagellin-sensitive 2 (FLS2). However, there are many differences in the interactions between various bacterial species and plants. For example, cooperation between Azospirillum spp. and rice cultivars depends on the genetic nature of both the bacterium and the plant (Drogue et al. 2014). Unlike previous studies, the present work revealed that the beneficial $B$. velezensis triggered an ETI defense program by activating a large number of resistance $(R)$ genes assigned to the NBS-LRR family. Moreover, we noticed that, after $G$. graminis var. tritici infection, roots from plants pretreated with B. velezensis elicited more drastic changes in important PRR genes triggered by PAMPs, such as brassinosteroid insensitive 1-associated kinase 1 (BAK1), FLS2, and chitin elicitor receptor kinase (CERK), which can transduce signals that trigger PTI (Dodds and Rathjen 2010). Also, B. velezensis-treated plants were able to rapidly activate the expression of $R$ genes involved in ETI when the plants were challenged by $G$. graminis var. tritici. These results suggest that precolonization by Bacillus spp. not only sensitizes the PTI but also the ETI for enhanced defense in wheat roots. Perhaps for this reason, the primed state induced by a PGPR strain is often invisible in unchallenged plants (Pieterse et al. 2014). Therefore, when the ISR-expressing wheat plants were challenged with the pathogen $G$. graminis var. tritici, larger transcriptional changes may have occurred than in nonprimed plants.

When compared with inoculation by a single microorganism, the results we obtained for combined inoculation (bacterium
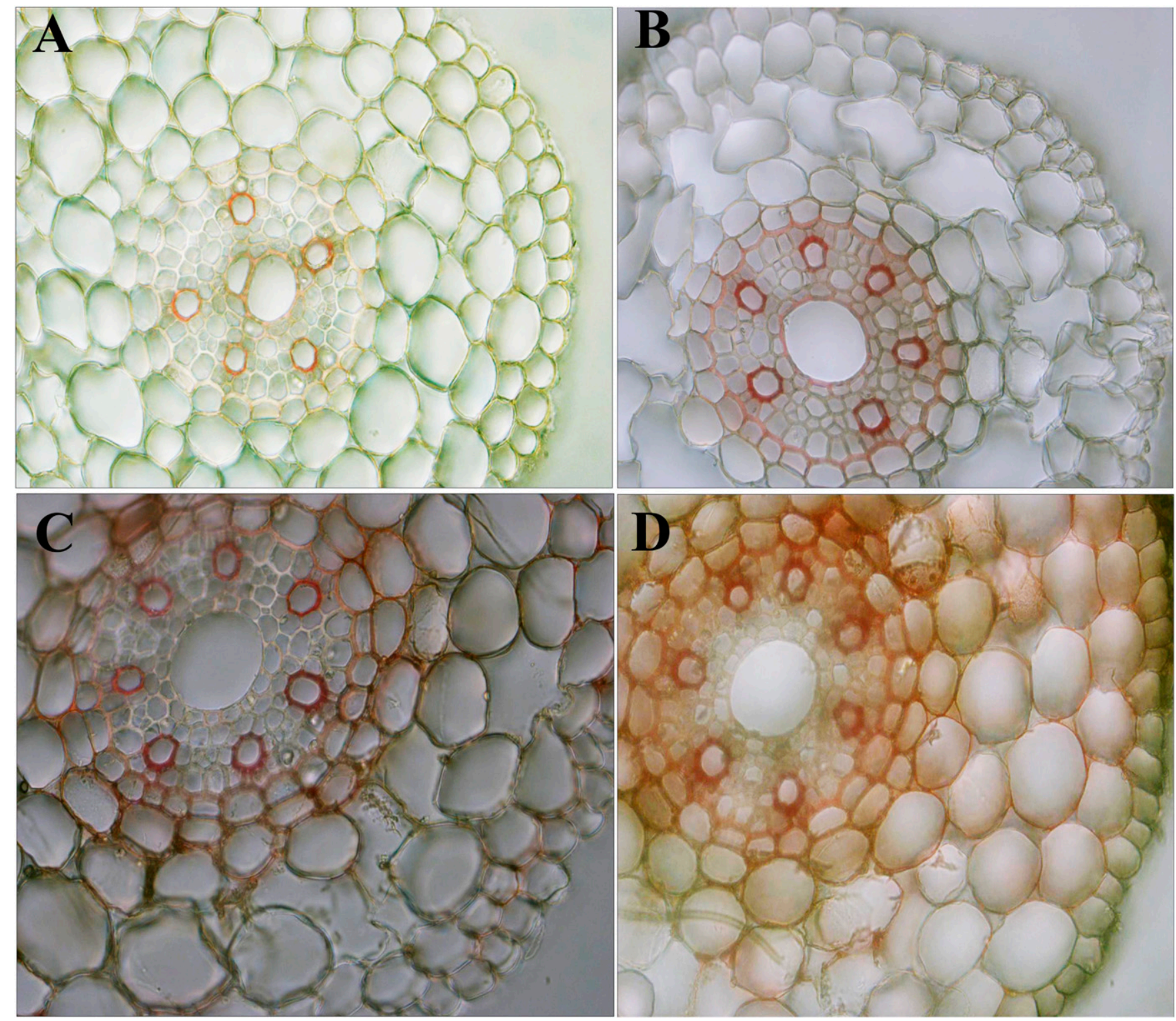

Fig. 6. Effects of lignin structure on wheat colonized by Bacillus velezensis, Gaeumannomyces graminis var. tritici, and their combination. Transverse sections of adventitious roots under different treatments. A, Control; B, B. velezensis; C, G. graminis var. tritici; and D, both organisms combined. The red stain indicates the location of lignin structure with phloroglucinol-HCl staining. 
plus fungus) are more likely to reflect the actual transcriptional and translational changes that might be expected under natural agriculture conditions. Furthermore, our work sheds light how wheat responds to beneficial and pathogenic microorganisms simultaneously. We found that the changes in gene expression elicited by combination treatment was more similar to that of the individual infection by G. graminis var. tritici (Figs. 1 and 6B). According to a recent study described by Kang et al. (2018), although inoculation of $B$. velezensis can alleviate wheat disease, it does not completely prevent infection by the pathogen G. graminis var. tritici. Therefore, like the pathogen infection individually, the combination of $B$. velezensis and $G$. graminis var. tritici ultimately gave rise to take-all disease (Kang et al. 2018). Such an outcome contributed to the similar expression pattern between G. graminis var. tritici and combination samples but it also indicated that, when wheat roots are challenged by both beneficial and pathogenic microorganisms, the plants most likely prioritize response mechanisms to resist the invasion of the pathogen.

Plants can activate defense responses to both pathogenic and beneficial microorganisms. These defense responses are largely modulated through hormone production. The plant hormones ET, JA, and SA are important regulators of induced defense mechanisms (Vos et al. 2015). Previous studies have found that treatment with ISR-inducing PGPR did not lead to upregulation of JA- or ET-responsive genes in plants; however, when challenged with pathogens, these plants exhibited induced expression of the JA-responsive gene PDF1.2 (Pieterse et al. 2014; Van Oosten et al. 2008), which suggests that pretreatment with the beneficial strain can prime plant immune system sensitivity. However, in this study, unlike other PGPR strains, colonization of B. velezensis promoted the accumulation of JA in wheat roots. Moreover, the marker gene PDF1.2 was not induced but was, instead, inhibited. In addition, when wheat was subsequently challenged with the pathogen $G$. graminis var. tritici, the degree of inhibition of PDF1.2 gene expression was aggravated compared with that during G. graminis var. tritici infection individually. These data appear to indicate that the colonization of $B$. velezensis caused the JA-induced resistance pathway to be inactivated. However, in the current study, six $P R l$ genes were all induced by $B$. velezensis treatment. $P R I$ is a marker gene for Arabidopsis SAR (Dong 2004), indicating that the SA-mediated SAR defense response was also triggered by B. velezensis. In addition, $B$. velezensis-pretreated plants rapidly accumulated SA and the expression of $P R I$ was slightly higher than for $G$. graminis var. tritici infection individually when the wheat plants were challenged with G. graminis var. tritici. Based on the current data, we suggest that, similar to ISR triggered by JA or $\mathrm{ET}$, B. velezensis-mediated SAR in wheat also requires sensitivity to SA but is not accompanied by an increase in its production (Pieterse et al. 2000; van Wees et al. 1999).

Compared with the control, G. graminis var. tritici infection led to a larger change in SA than JA accumulation. In addition, whereas the SA-responsive gene $P R I$ was induced, the JAresponsive gene $P D F 1.2$ was inhibited. Based on our evaluation of hormone content and the expression of resistance marker expression, we conclude that G. graminis var. tritici infection induces a stronger SA-mediated resistance response than that of JA, suggesting a more important role for SA than JA in the plant defense against $G$. graminis var. tritici infection. However, there are differences between our results and previous findings. SA-dependent defenses are generally effective against biotrophic pathogens, whereas JA-dependent defenses are generally effective against necrotrophic pathogens and herbivorous insects (Glazebrook 2005). According to our understanding, this principle is not applicable in all circumstances because many factors (i.e., ET, JA, and SA) contribute to sensitizing a plant's immune system. Furthermore, plants employ different strategies to resist infection by various pathogens, often utilizing the most efficient approach (Shigenaga et al. 2017; Vos et al. 2015). Selecting an effective defense response is generally determined by the genetic characteristics of the plants and the pathogens themselves (Robert-Seilaniantz et al. 2011).

Studies on the antagonistic relationship between SA- and JAsignaling pathways have focused on dicotyledonous plants, particularly the model species A. thaliana (Shigenaga et al. 2017; Vos et al. 2015). Overall, our results reveal that the antagonistic relationship between SA and JA in wheat is not affected by single or combined, beneficial or pathogenic microorganisms (Fig. 3; Table 2) but, instead, is enhanced or attenuated in response to different microorganisms. When wheat was coinfected with the beneficial B. velezensis and the pathogen G. graminis var. tritici, it further enhanced the SA-mediated signaling pathway and weakened the JA-mediated pathway. Furthermore, coinoculation of $B$. velezensis and G. graminis var. tritici resulted in changes in gene expression more similar to that of G. graminis var. tritici individually (Fig. 1). Therefore, in order to more effectively resist the pathogen $G$. graminis var. tritici, a more efficient approach might be chosen which, as this study suggests, is likely to involve the SA-mediated pathway. However, due to the antagonistic relationship between JA and SA, enhancement of the SA pathway will likely attenuate the JA pathway, which is viewed as a costsaving strategy that has evolved in plants to reduce allocation costs by repression of unnecessary defenses, thereby minimizing trade-offs between plant defense and growth (Vos et al. 2015).

Deposition of lignin has been shown to enhance plant resistance to infection by preventing the diffusion of invading pathogens (Bellincampi et al. 2014). Lignin is responsible for preventing cell-wall degradation by pathogen-produced enzymes and for limiting the diffusion of pathogen-produced toxins (Sattler and Funnell-Harris 2013). Generally, a positive correlation was found between lignin accumulation and the severity of disease caused by pathogen infection (Ma et al. 2018). Due to the biocontrol and induced wheat resistance properties of $B$. velezensis, the disease severity in wheat roots inoculated with both $B$. velezensis and $G$. graminis var. tritici became weaker than that of $G$. graminis var. tritici infection alone (Kang et al. 2018). However, the presence of B. velezensis further enhanced lignification that resulted from G. graminis var. tritici inoculation, which is thought to effectively protect adjacent plant cells from infection (Sattler and Funnell-Harris 2013). We suggest that the enhancement of lignin accumulation is caused by the additive effect of the beneficial bacteria $B$. velezensis and the pathogen G. graminis var. tritici. In addition, phenylpropanoid metabolism involves a complex series of branching biochemical pathways that provide plants with diverse compounds, many of which are used as structural cell components (lignin, suberin, and other cell-wall-associated phenolics), pigments (flavonoids and anthocyanins), immunity signals (SA), and toxins (coumarins and furanocoumarins) (Khanam et al. 2018; Vogt 2010). PAL is a key enzyme that catalyzes an early reaction in the production of phenylpropanoids. Therefore, the upregulation of PAL that we observed in the combination coinoculation experiment likely reflects the importance of this enzyme in the biosynthesis of not only SA but also lignin.

According to the strict criteria of $\geq 1.5$-fold cutoff and $P$ value $<$ 0.05 , only a few DEPs were identified in G. graminis var. tritici- or combination-colonized wheat roots, which might be due to the sensitivity of proteome analysis, availability of protein database, and posttranslational modifications of proteins (Pascovici et al. 2013). Through literature investigation, we found that there is no unified standard for the identification of DEPs. For example, Chen et al. (2016) used fold change and $P$ value to 
identify the DEPs in cotton roots and leaves under salt stress, while Wang et al. (2014) and Sun et al. (2016) used fold change and error factor to distinguish DEPs in rice roots under aluminum stress and different cultivars of ginseng, respectively. Zargar et al. (2015) and Wang et al. (2016) even used fold change only to identify the DEPs in A. thaliana and Triticum polonicum roots in response to metal deficiency based on iTRAQ analysis. In addition, although fold change is applied to proteome studies, the level of fold change also varies in different studies, such as 1.1 (Wang et al. 2014), 1.2 (Chen et al. 2016), 1.3 (Gao et al. 2016), 1.4 (Sun et al. 2016), 1.5 (Huang et al. 2013; Wang et al. 2016), and 2 (Zargar et al. 2015). Therefore, how to define significantly differentially expressed proteins is worth further exploration.

Consistent with many other studies, the correlations between transcriptome and proteome data are very low (0.0116 to 0.0927) in B. velezensis-, $G$. graminis var. tritici-, and combination-colonized wheat roots. It has been explained that the low correlations between transcriptome and proteome data are related to the complexity of the process from gene transcription to protein synthesis in wheat roots (Fournier et al. 2010; Gedeon and Bokes 2012; Huang et al. 2013). For example, Huang et al. (2013) used RNA-seq and iTRAQ to study the nitrogen starvation response of Synechocystis sp. PCC 6803, and found that the correlation coefficients $(r)$ of transcriptome and proteome data at 24 and $48 \mathrm{~h}$ were 0.04 and -0.001 , respectively. They attributed this result to possible posttranscriptional, translation, or posttranslational regulation of mRNA. In addition, the iTRAQ technology mainly relies on the ability to match peptide tandem mass spectra to sequences from the protein databases (Pascovici et al. 2013). Due to the large genome size (17 gb) and high proportion of repetitive sequences $(>80 \%)$, hexaploid wheat contains a complex and huge protein database. When comparing protein homology, it is easy to have ambiguous matching, which affects protein recognition and quantitative analysis. Thus, both biological reasons (e.g., pretranslational or posttranslational regulation of gene expression) and technical reasons (i.e., spectral mismatches in wheat's complex genome) may lead to only a small number of proteins identified by iTRAQ and a low correlation between proteomic results and transcriptional results.

\section{Conclusion.}

In this study, we make the first report of hexaploid wheat response to B. velezensis CC09, G. graminis var. tritici, and both microorganisms combined through the comparative analysis of transcriptome and proteome. We showed that the SA-dependent type of ISR is also triggered by beneficial B. velezensis, and the enhancement of SA-dependent defense response (i.e., lignin deposition) by the preinoculated $B$. velezensis is shown to reduce the level of induced JA-dependent defense against subsequent pathogen G. graminis var. tritici infection. In addition, wheat roots pretreated by $B$. velezensis not only exhibit larger transcriptional or translational programming than that of a single microorganism but also possess a more sensitive plant innate immunity system when the wheat roots were challenged with subsequent pathogen $G$. graminis var. tritici. In short, the current study provide a deeper understanding of the relationships in the hexaploid wheat-beneficial strainpathogen fungus, which may lead to new biocontrol methods and help alleviate wheat crop-related disease issues.

\section{MATERIALS AND METHODS}

Wheat, bacterium, and fungus.

The winter wheat cultivar Sumai 188, purchased from Jiangsu Academy of Agricultural Sciences, Nanjing, China, was used in this study. The endophytic biocontrol bacterium B. velezensis CC09 (strain deposit number CICC 24093; GenBank accession number CP015443) and take-all-causing pathogen G. graminis var. tritici were used in this study. B. velezensis was cultured in liquid Luria-Bertani medium at $37^{\circ} \mathrm{C}$ in a rotatory shaker $(200 \mathrm{rpm})$. After growth for $12 \mathrm{~h}$, sample inocula $\left(1 \times 10^{8}\right.$ $\mathrm{CFU} \mathrm{ml} \mathrm{m}^{-1}$ ) were centrifuged at 7,000 $\times \mathrm{g}$ for $5 \mathrm{~min}$, and resuspended in sterile distilled water. G. graminis var. tritici was cultured at $25^{\circ} \mathrm{C}$ on potato dextrose agar medium for 10 days.

\section{Evaluation of global responses to single and combined microorganisms in wheat roots.}

Wheat seeds were surface disinfected and germinated as described by Kang et al. (2018). The germinated wheat seeds were cultured on a sterilized raising plate containing $120 \mathrm{ml}$ of half-strength Murashige and Skoog medium (catalog number M0222.0050; Duchefa Biochemie) and incubated at $25^{\circ} \mathrm{C}$ under $14 \mathrm{~h}$ of light and $10 \mathrm{~h}$ of darkness for 7 days. Roots of wheat seedlings were inoculated with $30 \mathrm{ml}$ of B. velezensis inoculum or the same amount of sterile distilled water. Five days postinoculation with $B$. velezensis, the seedlings were placed on a water-agar plate and each root of the seedlings was inoculated with a plug of mycelia that was cut from the colony margin of G. graminis var. tritici. Nonpathogenized, nonbacterized, and watered control plants were also included. This experiment can be simply described as four treatments: (i) control (CK), roots inoculated with sterile water; (ii) B. velezensis, roots inoculated with beneficial bacterium $B$. velezensis only; (iii) G. graminis var. tritici, roots inoculated with take-all pathogen $G$. graminis var. tritici only; and (iv) combination, roots inoculated with both $B$. velezensis and $G$. graminis var. tritici. Seedlings were grown under a controlled environment (light intensity of $200 \mu \mathrm{mol} \mathrm{m} \mathrm{m}^{-2} \mathrm{~s}^{-1}$, cycle of $14 \mathrm{~h} /$ day and $10 \mathrm{~h} / \mathrm{night}$, at a constant temperature of $25^{\circ} \mathrm{C}$ and a relative humidity of $50 \%$ ). At 8 days after bacterial inoculation, approximately 30 seedlings were collected and stored at $-80^{\circ} \mathrm{C}$ prior to RNA or protein extraction. Fresh samples were used for biochemical assays, including the observation of lignin structure and determination of plant hormones. Each experiment was repeated three times, with 10 replicates per treatment.

\section{Microscopic observation of lignification of root tissue.}

Lignification is an indicator of plant defense against pathogens. To understand whether the combined inoculation of $B$. velezensis and $G$. graminis var. tritici can enhance the lignification of root tissue, we conducted histological analysis based on a previously described staining method with small modification (Zhu et al. 2018). Briefly, the cross section of the root samples was directly prepared by the bare-handed method. Then, a few drops of acidified phloroglucinol ethanol solution ( $2 \%$ phloroglucinol dissolved in ethanol, mixed with $1 \% \mathrm{HCl}$ ) were poured on the sections of the roots, and immediately observed with a light microscope (CX40; Shunyu).

\section{Quantification of phytohormone.}

A 1.0-g root sample frozen in liquid nitrogen was used for extraction and quantification of phytohormones JA and SA using the method described by Cheng et al. (2017). An enzymelinked immunosorbent assay kit was used to quantify the levels of the phytohormones JA and SA, purchased from Jiangsu Meibiao Biological Technology Company Limited. All measurements were replicated three times.

\section{Total RNA extraction and RNA-seq analysis.}

Roots of five plants were pooled for total RNA extraction using an RNAprep Pure Plant Kit (catalog number DP441; 
Tiangen) following the manufacturer's instructions. The purity and integrity of the obtained RNA were detected using an Agilent 2100 Bioanalyzer RNA chip (Agilent Technologies). In total, four RNA-seq libraries were constructed, representing the four treatments of CK, B. velezensis, G. graminis var. tritici, and combination, and sequenced on an Illumina HiSeqTM 4000 platform following a default Illumina stranded RNA protocol (Personalbio). Each library produced approximately 1,800 million 150-bp reads yielding more than $25 \mathrm{gb}$ of sequenced data so as to detect the low-abundance mRNA. Filtered clean reads were mapped to reference genome of T. aestivum (The International Wheat Sequencing Consortium [IWGSC] 2014) using SOAPaligner/SOAP2 software (Li et al. 2009), and deposited in the NCBI Sequence Read Archive under BioProject ID PRJNA485739. Transcript expression was normalized to RPKM for quantification of each gene. Genes exhibited significant changes in expression as determined through the DESeq R package (Love et al. 2014). The resulting $P$ values were adjusted using the Benjamini and Hochberg approach for controlling the false discovery rate. Genes with an adjusted $P$ value $<0.05$ found by DESeq and absolute value of $\log _{2}$ ratio $\geq 1$ were assigned as DEGs. The DEGs were annotated using the Kyoto Encyclopedia of Genes and Genomes (KEGG) database. STEM software was used to identify the significantly enriched expression patterns $(P$ value $<0.05)$ in $B$. velezensis-, $G$. graminis var. tritici-, and combination-colonized wheat roots. The RPKM value of CK, B. velezensis, G. graminis var. tritici, and the combination was used as the input dataset, and we performed the trend analysis to identify the significantly enriched expression patterns $(P$ value $<0.05)$ on the STEM platform. The parameters were set on the STEM platform, including the input of $\log _{2}$-transformed RPKM values, a maximum number of model profiles of 20 , and a maximum unit change of 2 .

\section{Total protein extraction and iTRAQ analysis.}

A $0.5-\mathrm{g}$ sample of root tissue from each treatment was ground in liquid nitrogen and extracted using $4 \mathrm{ml}$ of extraction buffer $(50 \mathrm{mM}$ Tris- $\mathrm{HCl}$ [pH 7.4], $100 \mathrm{mM} \mathrm{NaCl}$, $1 \mathrm{mM}$ phenylmethylsulfonyl fluoride, $1 \mathrm{mM}$ EDTA, $1 \%$ Triton X-100, 1\% sodium deoxycholate, and 2\% sodium dodecyl sulfate) following the method described by Wang et al. (2014), with minor modification. The mixture was purified using acetone, and the purified proteins were dissolved in a 7M urea solution and quantified using a QuantiPro BCA Assay Kit (TaKaRa Bio Inc.) following the manufacturer's instructions. The obtained proteins were stored at $-80^{\circ} \mathrm{C}$ for iTRAQ analysis, including iTRAQ labeling, SCX chromatography fractionation, and liquid chromatographyelectrospray ionization tandem mass spectrometry (MS/MS) analysis with a 5600 TripleTOF platform (AB SCIEX) following the method described by Yang et al. (2013). The MS/MS data were analyzed through a search and quantitative analysis against the nonredundant protein database of the hexaploid wheat genome (The International Wheat Sequencing Consortium [IWGSC] 2014) using ProteinPilot Software v4.5 (AB SCIEX). Proteins were identified using the following parameters: sample type = iTRAQ 4-plex (peptide-labeled), Cys; alkylation = iodoacetamide ; and digestion $=$ trypsin. The MS proteomics data have been deposited at the iProx, which is an official member of ProteomeXchange Consortium, with the data set identifier IPX0001525003/PXD012900. An Unused Protein Score of 1.3 or more (equivalent to $95 \%$ confidence level) was used as threshold for a reliable protein identification. A 1.5-fold cutoff and a $P$ value $<0.05$ was set to identify the significant changes in abundance for the regulated proteins. The functional annotation and classification of all DEPs were also performed using the KEGG database.

\section{qRT-PCR validation.}

Total RNA was extracted using the method described above. First-strand complementary DNAs were synthesized using a PrimeScript first-strand cDNA synthesis kit (TaKaRa). PCR amplification of 18 selected DEGs of the given colonized wheat was performed with an Applied Biosystems 7500 Real-time PCR system (Applied Biosystems) using SYBR AdvantageqPCR Premix (Toyobo). Primers used for qRT-PCR amplification targeting the selected genes are listed in Supplementary Table S7. A fragment of the $\alpha$-tubulin gene (GenBank accession number U76558) was amplified with specific primers TUBF (5'ACC GCC AGC TCT TCC ACC CT-3') and TUBR (5'-TCA CTG GGG CAT AGG AGG AA- $3^{\prime}$ ) as the internal control for qRT-PCR analysis. A reaction mixture $(20 \mu \mathrm{l})$ consisted of $10 \mu \mathrm{l}$ of $2 \times$ SYBR Green PCR Master mix, $0.4 \mu \mathrm{l}$ of each primer $(10 \mu \mathrm{M}), 2 \mu \mathrm{l}$ of $\mathrm{cDNA}$, and $7.2 \mu \mathrm{l}$ of nuclease-free water. qPCR conditions comprised one cycle at $95^{\circ} \mathrm{C}$ for $10 \mathrm{~s}$, followed by 40 cycles at $95^{\circ} \mathrm{C}$ for $4 \mathrm{~s}, 58^{\circ} \mathrm{C}$ for $15 \mathrm{~s}$, and $72^{\circ} \mathrm{C}$ for $34 \mathrm{~s}$. Melting curve analysis and electrophoresis were performed to verify the specificity and identity of the qRT-PCR products. In total, nine replicates were used for each sample (three biological replicates $\times$ three technical replicates). Gene expression was evaluated by the $2^{-\Delta \Delta \mathrm{Ct}}$ method (Livak and Schmittgen 2001).

\section{Statistical analysis.}

The phytohormone data between treatments were subjected to analysis of variance and considered statistically significant at $P$ value $<0.05$ according to Fisher's least significant difference test. GraphPad Prism software (version 3.02) or R (version 3.5.0) was used to prepare figures.

\section{ACKNOWLEDGMENTS}

We thank the State Key Laboratory of Pharmaceutical Biotechnology, School of Life Sciences, Nanjing University for providing instruments.

\section{AUTHOR-RECOMMENDED INTERNET RESOURCES}

IWGSC index: https://urgi.versailles.inra.fr/download/iwgsc Kyoto Encyclopedia of Genes and Genomes database: https://www.kegg.jp Integrated Proteome Resources database: https://www.iprox.org

\section{LITERATURE CITED}

Bano, A., and Muqarab, R. 2017. Plant defence induced by PGPR against Spodoptera litura in tomato (Solanum lycopersicum L.). Plant Biol. 19: 406-412.

Bellincampi, D., Cervone, F., and Lionetti, V. 2014. Plant cell wall dynamics and wall-related susceptibility in plant-pathogen interactions. Front. Plant Sci. 5:228.

Berrocal-Lobo, M., and Molina, A. 2008. Arabidopsis defense response against Fusarium oxysporum. Trends Plant Sci. 13:145-150.

Bithell, S. L., McKay, A. C., Butler, R. C., and Cromey, M. G. 2016. Consecutive wheat sequences: Effects of contrasting growing seasons on concentrations of Gaeumannomyces graminis var. tritici DNA in soil and take-all disease across different cropping sequences. J. Agric. Sci. 154: 472-486.

Cai, X., Kang, X., Xi, H., Liu, C., and Xue, Y. 2016. Complete genome sequence of the endophytic biocontrol strain Bacillus velezensis CC09. Genome Announce. 4:e01048-16.

Cai, X.-C., Liu, C.-H., Wang, B.-T., and Xue, Y.-R. 2017. Genomic and metabolic traits endow Bacillus velezensis $\mathrm{CC} 09$ with a potential biocontrol agent in control of wheat powdery mildew disease. Microbiol. Res. 196:89-94.

Chen, T., Zhang, L., Shang, H., Liu, S., Peng, J., Gong, W., Shi, Y., Zhang, S., Li, J., Gong, J., Ge, Q., Liu, A., Ma, H., Zhao, X., and Yuan, Y. 2016. 
iTRAQ-based quantitative proteomic analysis of cotton roots and leaves reveals pathways associated with salt stress. PLoS One 11 : e0148487.

Chen, Y. C., Holmes, E. C., Rajniak, J., Kim, J. G., Tang, S., Fischer, C. R., Mudgett, M. B., and Sattely, E. S. 2018. $N$-hydroxy-pipecolic acid is a mobile metabolite that induces systemic disease resistance in Arabidopsis. Proc. Natl. Acad. Sci. U.S.A. 115:E4920-E4929.

Cheng, Y. Q., Zhao, Y. X., Liu, J. F., Yang, B., and Ming, Y. T. 2017. Comparison of phytohormone biosynthesis and signal transduction pathways in developing and abortive hazelnut ovules. Plant Growth Regul. 81:147-157.

Cook, R. J. 2003. Take-all of wheat. Physiol. Mol. Plant Pathol. 62:73-86.

Daval, S., Lebreton, L., Gazengel, K., Boutin, M., Guillerm-Erckelboudt, A. Y., and Sarniguet, A. 2011. The biocontrol bacterium Pseudomonas fluorescens Pf29Arp strain affects the pathogenesis-related gene expression of the take-all fungus Gaeumannomyces graminis var. tritici on wheat roots. Mol. Plant Pathol. 12:839-854.

Dodds, P. N., and Rathjen, J. P. 2010. Plant immunity: Towards an integrated view of plant-pathogen interactions. Nat. Rev. Genet. 11:539-548.

Dong, X. 2004. NPR1, all things considered. Curr. Opin. Plant Biol. 7: 547-552.

Drogue, B., Sanguin, H., Chamam, A., Mozar, M., Llauro, C., Panaud, O., Prigent-Combaret, C., Picault, N., and Wisniewski-Dyé, F. 2014. Plant root transcriptome profiling reveals a strain-dependent response during Azospirillum-rice cooperation. Front. Plant Sci. 5:607.

Duba, A., Goriewa-Duba, K., and Wachowska, U. 2018. A review of the interactions between wheat and wheat pathogens: Zymoseptoria tritici, Fusarium spp. and Parastagonospora nodorum. Int. J. Mol. Sci. 19:1138.

Engelmann, I., Griffon, A., Tichit, L., Montañana-Sanchis, F., Wang, G. Reinke, V., Waterston, R. H., Hillier, L. W., and Ewbank, J. J. 2011. A comprehensive analysis of gene expression changes provoked by bacterial and fungal infection in C. elegans. PLoS One 6:e19055.

Erayman, M., Turktas, M., Akdogan, G., Gurkok, T., Inal, B., Ishakoglu, E., Ilhan, E., and Unver, T. 2015. Transcriptome analysis of wheat inoculated with Fusarium graminearum. Front. Plant Sci. 6:867.

Fournier, M. L., Paulson, A., Pavelka, N., Mosley, A. L., Gaudenz, K., Bradford, W. D., Glynn, E., Li, H., Sardiu, M. E., Fleharty, B., Seidel, C., Florens, L., and Washburn, M. P. 2010. Delayed correlation of mRNA and protein expression in rapamycin-treated cells and a role for $\mathrm{Ggc} 1$ in cellular sensitivity to rapamycin. Mol. Cell. Proteomics 9:271-284.

Gao, Y., Lim, T. K., Lin, Q., and Li, S. F. 2016. Identification of cypermethrin induced protein changes in green algae by iTRAQ quantitative proteomics. J. Proteomics 139:67-76.

Gedeon, T., and Bokes, P. 2012. Delayed protein synthesis reduces the correlation between mRNA and protein fluctuations. Biophys. J. 103: 377-385.

Glazebrook, J. 2005. Contrasting mechanisms of defense against biotrophic and necrotrophic pathogens. Annu. Rev. Phytopathol. 43:205-227.

Gond, S. K., Bergen, M. S., Torres, M. S., White, J. F., and Kharwar, R. N. 2015. Effect of bacterial endophyte on expression of defense genes in Indian popcorn against Fusarium moniliforme. Symbiosis 66:133-140.

Gonzalez, E., Pitre, F. E., Pagé, A. P., Marleau, J., Guidi Nissim, W., StArnaud, M., Labrecque, M., Joly, S., Yergeau, E., and Brereton, N. J. B. 2018. Trees, fungi and bacteria: Tripartite metatranscriptomics of a root microbiome responding to soil contamination. Microbiome 6:53.

Guilleroux, M., and Osbourn, A. 2004. Gene expression during infection of wheat roots by the 'take-all' fungus Gaeumannomyces graminis. Mol Plant Pathol. 5:203-216.

Hartmann, M., Zeier, T., Bernsdorff, F., Reichel-Deland, V., Kim, D., Hohmann, M., Scholten, N., Schuck, S., Bräutigam, A., Hölzel, T., Ganter, C., and Zeier, J. 2018. Flavin monooxygenase-generated Nhydroxypipecolic acid is a critical element of plant systemic immunity. Cell 173:456-469.e16.

Huang, S., Chen, L., Te, R., Qiao, J., Wang, J., and Zhang, W. 2013. Complementary iTRAQ proteomics and RNA-seq transcriptomics reveal multiple levels of regulation in response to nitrogen starvation in Synechocystis sp. PCC 6803. Mol. Biosyst. 9:2565-2574.

Jain, S., Choudhary, D. K., Sharma, K. P., and Aggarwal, R. 2018. Bacterial mediated plant protection: Induced systemic resistance in soybean. Pages 193-206 in: Microbial Biotechnology. J. Patra, G. Das, and H. S. Shin, eds. Springer, Singapore.

Kang, X. X., Zhang, W. L., Cai, X. C., Zhu, T., Xue, Y. R., and Liu, C. H. 2018. Bacillus velezensis CC09: A potential 'vaccine' for controlling wheat diseases. Mol. Plant-Microbe Interact. 31:623-632.

Khanam, S., Bauters, L., Singh, R. R., Verbeek, R., Haeck, A., Sultan, S. M. D., Demeestere, K., Kyndt, T., and Gheysen, G. 2018. Mechanisms of resistance in the rice cultivar Manikpukha to the rice stem nematode Ditylenchus angustus. Mol. Plant Pathol. 19:1391-1402.
Klessig, D. F., Choi, H. W., and Dempsey, D. A. 2018. Systemic acquired resistance and salicylic acid: Past, present, and future. Mol. PlantMicrobe Interact. 31:871-888.

Lakshmanan, V., Castaneda, R., Rudrappa, T., and Bais, H. P. 2013. Root transcriptome analysis of Arabidopsis thaliana exposed to beneficial Bacillus subtilis FB17 rhizobacteria revealed genes for bacterial recruitment and plant defense independent of malate efflux. Planta 238:657-668.

Li, R., Yu, C., Li, Y., Lam, T. W., Yiu, S. M., Kristiansen, K., and Wang, J. 2009. SOAP2: An improved ultrafast tool for short read alignment. Bioinformatics 25:1966-1967.

Livak, K. J., and Schmittgen, T. D. 2001. Analysis of relative gene expression data using real-time quantitative PCR and the $2^{-\Delta \Delta \mathrm{Ct}}$ method. Methods 25:402-408.

Lopez-Gomez, M., Sandal, N., Stougaard, J., and Boller, T. 2012. Interplay of flg22-induced defence responses and nodulation in Lotus japonicus J. Exp. Bot. 63:393-401.

Love, M., Anders, S., and Huber, W. 2014. Differential analysis of count data-the DESeq2 package. Genome Biol. 15:550.

Ma, Q. H., Zhu, H. H., and Qiao, M. Y. 2018. Contribution of both lignin content and sinapyl monomer to disease resistance in tobacco. Plant Pathol. 67:642-650.

Mhlongo, M. I., Piater, L. A., Madala, N. E., Labuschagne, N., and Dubery, I. A. 2018. The chemistry of plant-microbe interactions in the rhizosphere and the potential for metabolomics to reveal signaling related to defense priming and induced systemic resistance. Front. Plant Sci. 9:112.

Millet, Y. A., Danna, C. H., Clay, N. K., Songnuan, W., Simon, M. D., Werck-Reichhart, D., and Ausubel, F. M. 2010. Innate immune responses activated in Arabidopsis roots by microbe-associated molecular patterns. Plant Cell 22:973-990.

Morris, W. F., Traw, M. B., and Bergelson, J. 2006. On testing for a tradeoff between constitutive and induced resistance. Oikos 112:102-110.

Pascovici, D., Gardiner, D. M., Song, X., Breen, E., Solomon, P. S., Keighley, T., and Molloy, M. P. 2013. Coverage and consistency: Bioinformatics aspects of the analysis of multirun iTRAQ experiments with wheat leaves. J. Proteome Res. 12:4870-4881.

Peng, F. Y., and Yang, R. C. 2017. Prediction and analysis of three gene families related to leaf rust (Puccinia triticina) resistance in wheat (Triticum aestivum L.). BMC Plant Biol. 17:108.

Piasecka, A., Jedrzejczak-Rey, N., and Bednarek, P. 2015. Secondary metabolites in plant innate immunity: Conserved function of divergent chemicals. New Phytol. 206:948-964.

Pieterse, C. M. J., Van Pelt, J. A., Ton, J., Parchmann, S., Mueller, M. J., Buchala, A. J., Métraux, J., and Van Loon, L. C. 2000. Rhizobacteriamediated induced systemic resistance (ISR) in Arabidopsis requires sensitivity to jasmonate and ethylene but is not accompanied by an increase in their production. Physiol. Mol. Plant Pathol. 57:123-134.

Pieterse, C. M. J., Zamioudis, C., Berendsen, R. L., Weller, D. M., Van Wees, S. C., and Bakker, P. A. H. M. 2014. Induced systemic resistance by beneficial microbes. Annu. Rev. Phytopathol. 52:347-375.

Puga-Freitas, R., Belkacem, L., Barot, S., Bertrand, M., Roger-Estrade, J., and Blouin, M. 2017. Transcriptional profiling of wheat in response to take-all disease and mechanisms involved in earthworm's biocontrol effect. Eur. J. Plant Pathol. 144:1-11.

Robert-Seilaniantz, A., Grant, M., and Jones, J. D. G. 2011. Hormone crosstalk in plant disease and defense: More than just jasmonatesalicylate antagonism. Annu. Rev. Phytopathol. 49:317-343.

Saia, S., Rappa, V., Ruisi, P., Abenavoli, M. R., Sunseri, F., Giambalvo, D. Frenda, A. S., and Martinelli, F. 2015. Soil inoculation with symbiotic microorganisms promotes plant growth and nutrient transporter genes expression in durum wheat. Front. Plant Sci. 6:815.

Sattler, S. E., and Funnell-Harris, D. L. 2013. Modifying lignin to improve bioenergy feedstocks: Strengthening the barrier against pathogens? Front. Plant Sci. 4:70.

Shigenaga, A. M., Berens, M. L., Tsuda, K., and Argueso, C. T. 2017. Towards engineering of hormonal crosstalk in plant immunity. Curr. Opin. Plant Biol. 38:164-172.

Singh, A. A., Fatima, A., Mishra, A. K., Chaudhary, N., Mukherjee, A., Agrawal, M., and Agrawal, S. B. 2018. Assessment of ozone toxicity among 14 Indian wheat cultivars under field conditions: Growth and productivity. Environ. Monit. Assess. 190:190.

Singh, R. P., Hodson, D. P., Huerta-Espino, J., Jin, Y., Bhavani, S., Njau, P., Herrera-Foessel, S., Singh, P. K., Singh, S., and Govindan, V. 2011. The emergence of Ug99 races of the stem rust fungus is a threat to world wheat production. Annu. Rev. Phytopathol. 49:465-481.

Souza, E. M., Chubatsu, L. S., Huergo, L. F., Monteiro, R., Camilios-Neto, D., Wassem, R., and de Oliveira Pedrosa, F. 2014. Use of nitrogen-fixing bacteria to improve agricultural productivity. BMC Proc. 8:023. 
Spoel, S. H., and Dong, X. 2012. How do plants achieve immunity? Defence without specialized immune cells. Nat. Rev. Immunol. 12:89-100.

Srivastava, S., Bist, V., Srivastava, S., Singh, P. C., Trivedi, P. K., Asif, M. H., Chauhan, P. S., and Nautiyal, C. S. 2016. Unraveling aspects of Bacillus amyloliquefaciens mediated enhanced production of rice under biotic stress of Rhizoctonia solani. Front. Plant Sci. 7:587.

Stringlis, I. A., Proietti, S., Hickman, R., Van Verk, M. C., Zamioudis, C., and Pieterse, C. M. J. 2018. Root transcriptional dynamics induced by beneficial rhizobacteria and microbial immune elicitors reveal signatures of adaptation to mutualists. Plant J. 93:166-180.

Sun, H., Liu, F., Sun, L., Liu, J., Wang, M., Chen, X., Xu, X., Ma, R., Feng, K., and Jiang, R. 2016. Proteomic analysis of amino acid metabolism differences between wild and cultivated Panax ginseng. J. Ginseng Res. 40:113-120.

The International Wheat Genome Sequencing Consortium (IWGSC). 2014. A chromosome-based draft sequence of the hexaploid bread wheat (Triticum aestivum) genome. Science 345:1251788.

Trdá, L., Fernandez, O., Boutrot, F., Héloir, M. C., Kelloniemi, J., Daire, X., Adrian, M., Clément, C., Zipfel, C., Dorey, S., and Poinssot, B. 2014. The grapevine flagellin receptor VvFLS2 differentially recognizes flagellin-derived epitopes from the endophytic growth-promoting bacterium Burkholderia phytofirmans and plant pathogenic bacteria. New Phytol. 201:1371-1384.

Van Oosten, V. R., Bodenhausen, N., Reymond, P., Van Pelt, J. A., Van Loon, L. C., Dicke, M., and Pieterse, C. M. 2008. Differential effectiveness of microbially induced resistance against herbivorous insects in Arabidopsis. Mol. Plant-Microbe Interact. 21:919-930.

van Wees, S. C., Luijendijk, M., Smoorenburg, I., van Loon, L. C., and Pieterse, C. M. 1999. Rhizobacteria-mediated induced systemic resistance (ISR) in Arabidopsis is not associated with a direct effect on expression of known defense-related genes but stimulates the expression of the jasmonateinducible gene Atvsp upon challenge. Plant Mol. Biol. 41:537-549.

Verhagen, B. W., Glazebrook, J., Zhu, T., Chang, H. S., van Loon, L. C., and Pieterse, C. M. 2004. The transcriptome of rhizobacteria-induced systemic resistance in Arabidopsis. Mol. Plant-Microbe Interact. 17:895-908.

Vogt, T. 2010. Phenylpropanoid biosynthesis. Mol. Plant 3:2-20.

Vos, I. A., Moritz, L., Pieterse, C. M., and Van Wees, S. C. 2015. Impact of hormonal crosstalk on plant resistance and fitness under multi-attacker conditions. Front. Plant Sci. 6:639.
Wang, Y., Wang, X., Wang, C., Wang, R., Peng, F., Xiao, X., Zeng, J., Fan, X., Kang, H., Sha, L., Zhang, H., and Zhou, Y. 2016. Proteomic profiling of the interactions of $\mathrm{Cd} / \mathrm{Zn}$ in the roots of dwarf polish wheat (Triticum polonicum L.). Front. Plant Sci. 7:1378.

Wang, Z. Q., Xu, X. Y., Gong, Q. Q., Xie, C., Fan, W., Yang, J. L., Lin, Q. S., and Zheng, S. J. 2014. Root proteome of rice studied by iTRAQ provides integrated insight into aluminum stress tolerance mechanisms in plants. J. Proteomics 98:189-205.

Williamson, B., Tudzynski, B., Tudzynski, P., and van Kan, J. A. L. 2007. Botrytis cinerea: The cause of grey mould disease. Mol. Plant Pathol. 8:561-580.

Xin, M., Wang, X., Peng, H., Yao, Y., Xie, C., Han, Y., Ni, Z., and Sun, Q. 2012. Transcriptome comparison of susceptible and resistant wheat in response to powdery mildew infection. Genomics Proteomics Bioinf. 10: 94-106.

Yang, L., Quan, X., Xue, B., Goodwin, P. H., Lu, S., Wang, J., Du, W., and $\mathrm{Wu}, \mathrm{C}$. 2015a. Isolation and identification of Bacillus subtilis strain YB-05 and its antifungal substances showing antagonism against Gaeumannomyces graminis var. tritici. Biol. Control 85:52-58.

Yang, L., Xie, L., Xue, B., Goodwin, P. H., Quan, X., Zheng, C., Liu, T., Lei, Z., Yang, X., Chao, Y., and Wu, C. 2015b. Comparative transcriptome profiling of the early infection of wheat roots by Gaeumannomyces graminis var. tritici. PLoS One 10:e0120691.

Yang, L. T., Qi, Y. P., Lu, Y. B., Guo, P., Sang, W., Feng, H., Zhang, H. X., and Chen, L. S. 2013. iTRAQ protein profile analysis of Citrus sinensis roots in response to long-term boron-deficiency. J. Proteomics 93: 179-206.

Zargar, S. M., Fujiwara, M., Inaba, S., Kobayashi, M., Kurata, R., Ogata, Y., and Fukao, Y. 2015. Correlation analysis of proteins responsive to $\mathrm{Zn}$, Mn, or Fe deficiency in Arabidopsis roots based on iTRAQ analysis. Plant Cell Rep. 34:157-166.

Zhang, L., Li, M., Li, Q., Chen, C., Qu, M., Li, M., Wang, Y., and Shen, X. 2018. The catabolite repressor/activator Cra is a bridge connecting carbon metabolism and host colonization in the plant drought resistancepromoting bacterium Pantoea alhagi LTYR-11Z. Appl. Environ. Microbiol. 84:e00054-18.

Zhu, N., Wu, D., and Chen, K. 2018. Label-free visualization of fruit lignification: Raman molecular imaging of loquat lignified cells. Plant Methods 14:58. 